\title{
Impactos do afastamento social na qualidade de vida de atletas de voleibol feminino de base durante a pandemia covid-19
}

\section{Impacts of social removal on the quality of life of female volleyball young athletes during the covid-19 pandemic}

DOI: $10.46919 / \operatorname{archv2n3-011~}$

Recebimento dos originais: 01/01/2021

Aceitação para publicação: 31/03/2021

\author{
Elias Rafael Hauschild \\ Acadêmico do curso de Educação Física Bacharelado, Universidade do Vale do Taquari - Univates, \\ E-mail: erhauschild@universo.univates.br \\ Rodrigo Lara Rother \\ Prof. Dr. do curso de Educação Física Bacharelado, Universidade do Vale do Taquari - Univates, \\ E-mail: rodrigorother@univates.br
}

\section{RESUMO}

A prática esportiva para crianças e adolescentes não se resume aos benefícios gerados para a saúde física, mas também social e psicológica, impactando diretamente na Qualidade de Vida (QV). Durante a pandemia, atletas jovens ingressaram em sessões online, treinando em suas casas, mas sem a convivência presencial com colegas, professores e adversários. Objetivo: Analisar os níveis de QV percebidos por atletas de voleibol feminino de base durante diferentes momentos do período de isolamento social e compará-los quanto a suas dimensões física, psicológica, social e ambiental. Metodologia: A partir do banco de dados de uma equipe de voleibol feminino, foram extraídos os resultados do questionário WHOQOL-bref para avaliações de QV das 25 atletas das equipes de competição femininas de base, com média de idade de 16,2 anos. As informações foram coletadas de abril a agosto de 2020, período em que não houveram aulas presenciais nas escolas, tampouco treinos presenciais da equipe. Também foi aplicado um segundo questionário indagando o tempo e o rigor do afastamento adotado pelas atletas. Resultados: Não foram encontradas diferenças significativa nos escores de QV quando comparados os diferentes meses da pandemia. A média foi classificada como "Boa" para todas as dimensões, sendo que a Psicológica obteve menor média, com 66,2 pontos. Já a maior foi para a Ambiental, com média de 76,4 pontos. Quanto ao tempo de afastamento, foram 163,8 dias em média. Quanto à classificação do rigor do afastamento social adotado, para uma escala de 1 a 5, as médias das respostas foram: Abril (3,7), Maio (4), Junho (3,5), Julho (3,2) e Agosto (3,4). Observou-se que as atletas mais velhas apresentaram um rigor de afastamento menor que as mais jovens, com ênfase nos meses de junho, julho e agosto. Ainda assim, foi encontrada correlação significativa entre um maior rigor de afastamento social e um menor nível de QV Geral e Físico. Conclusão: A QV avaliada manteve-se boa e estável durante o afastamento social ocorrido de abril a agosto. O baixo rigor do afastamento e a continuidade na realização de treinamentos online durante o período podem ser variáveis que auxiliaram na manutenção de níveis bons de QV. Mais estudos sobre os efeitos da prática de treinamentos em casa merecem atenção, já que foi uma prática comum para atletas durante a pandemia.

Palavras chave: Covid-19, Voleibol, Atletas jovens.

\begin{abstract}
The practice of sports for children and adolescents is not limited to the benefits generated for physical health, but also social and psychological, impacting directly on Quality of Life (QoL). During the pandemic, young athletes joined online sessions, training at their homes, but without the face-to-face contact with
\end{abstract}


peers, teachers, and opponents. Objective: To analyze the levels of QoL perceived by female volleyball athletes during different moments of the social isolation period and compare them as to their physical, psychological, social, and environmental dimensions. Methodology: From the database of a women's volleyball team, the results of the WHOQOL-bref questionnaire were extracted for QL assessments of the 25 athletes of the women's grassroots competition teams, with a mean age of 16.2 years. The information was collected from April to August 2020, a period in which there were no on-site classes at the schools, nor on-site team training sessions. A second questionnaire was also applied inquiring about the time and severity of the withdrawal adopted by the athletes. Results: No significant differences were found in QL scores when comparing the different months of the pandemic. The average was classified as "Good" for all dimensions, with the Psychological one having the lowest average, with 66.2 points. The highest was for the Environmental dimension, with an average of 76.4 points. The average time away from work was 163.8 days. As for the classification of the severity of the social withdrawal adopted, for a scale of 1 to 5, the averages of the answers were: April (3.7), May (4), June (3.5), July (3.2) and August (3.4). It was observed that the older athletes presented a lower withdrawal rigor than the younger ones, with emphasis on the months of June, July, and August. Even so, a significant correlation was found between a higher social withdrawal severity and a lower level of General and Physical QL. Conclusion: The QL assessed remained good and stable during the social withdrawal that occurred from April to August. The low severity of the withdrawal and the continuity of online training during the period may be variables that helped maintain good levels of QOL. Further studies on the effects of training at home deserve attention, as it was a common practice for athletes during the pandemic.

Keywords: Covid-19, Volleyball, Young athletes.

\section{INTRODUÇÃO}

A Organização Mundial da Saúde (OMS, 2020) define SARS-Cov-2 como uma nova cepa (tipo) de vírus que pertence à família de coronavírus (HCoVs). O COVID-19, como ficou conhecido, não havia sido identificado antes em seres humanos. É uma doença respiratória nova, de fácil contágio e, embora seus sintomas sejam semelhantes a uma gripe, os casos graves necessitam de internação hospitalar, auxílio de ventilação mecânica e a taxa de mortalidade é de 40 mortes para cada 100.000 contágios e sua taxa de letalidade é de 2,9\% (OPAS, 2020). Por ser nova, até o momento não há vacina, tampouco tratamento eficaz comprovado. Desta forma, a maneira mais eficiente de combater a doença é o afastamento social e bons hábitos de higiene combinados com utilização de equipamentos de proteção (ZHU, 2020).

Segundo Moura et al (2020), o desconhecimento de muitas informações sobre esta nova doença impactou em escala mundial todos os segmentos. Inúmeros tipos de atividades foram interrompidas, sejam profissionais, de lazer e até mesmo alguns serviços essenciais foram afetados. Entre as atividades fortemente impactadas, a prática esportiva é uma delas, resultando inclusive no adiamento dos Jogos Olímpicos de Tóquio/2020. Com a obrigatoriedade do afastamento social, muitos atletas dos mais diversos níveis e idades foram impedidos de realizar seus treinamentos. O impacto econômico gerado afetou também o apoio de patrocinadores, bolsas governamentais, salários de atletas e comissão técnica, cancelamento de eventos, entre outros. Se os problemas são grandes para atletas profissionais, os impactos podem ser ainda maiores nas categorias de base. 
No esporte de base existe a dimensão competitiva, de treinamento e disputa de eventos, mas além dela há também a dimensão formativa, social e voltada para a promoção saúde e Qualidade de Vida (QV) das crianças e adolescentes praticantes. Ressaltando a importância da prática esportiva para a saúde, Carazzato (1995) descreve alguns ótimos resultados fisiológicos da prática esportiva: hipertrofia e maior eficiência do músculo cardíaco, eliminação de gordura excedente pelo sistema endócrino e o controle dos níveis de insulina no sangue. Além disso, Benetti, Schneider e Meyer (2005) destacam que indivíduos jovens e saudáveis, praticantes de treinamento físico, têm benefícios relacionados ao crescimento.

Já quanto à QV, Simões (2011) investigou a percepção de QV em atletas de voleibol de diferentes idades, percebendo um alto nível de satisfação geral, além de ter identificado que os aspectos físicos e psicológicos são os que exercem maior influência na percepção de QV. Em outro estudo, Rother (2017) indica que o nível de QV das atletas gaúchas são bons e que os técnicos têm grande responsabilidade por manter ou melhorar estes resultados, seja com um bom planejamento de aplicação da carga de treinamento, controle do números de jogos e competições disputadas e promovendo cuidados de prevenção de lesões. O Conselho Regional de Educação Física (2020) afirma que as habilidades pessoais, motoras e as necessidades sociais das crianças e adolescentes devem ser desenvolvidas através do esporte.

Pela impossibilidade de realizar atividades presenciais, muitos tipos de serviços voltados à saúde, incluindo o treinamento esportivo, aderiram ao formato online durante a pandemia. Um recente estudo de Tenforde et al (2020) avaliou a satisfação do atendimento online de telereabilitação prestada por fisioterapia, fonoaudiologia e terapia ocupacional. Num total de 205 pacientes respondentes, 54\% responderam que, de forma geral, os resultados foram muito positivos, classificando-os como "excelentes" ou "muito bons". O treinamento de atletas seguiu a mesma direção, utilizando em muitos casos videoconferências e realização de atividades online, com os atletas exercitando-se em suas próprias casas.

Cientes da importância da prática de atividade física por estes jovens atletas, mesmo com a opção de realizá-las em casa, compreende-se que o impedimento de treinar presencialmente, interagindo com colegas de equipe, utilizando a estrutura do clube e com orientação in loco da comissão técnica pode afetar negativamente o desempenho esportivo e também a saúde e QV, além é claro de ser muito importante o convívio social neste faixa etária. Como vivemos um momento ímpar para a sociedade mas que pode repetir-se num futuro próximo, é importante investigar os efeitos da pandemia em atletas jovens, contribuindo com novos conhecimentos que auxiliem na minimização de prejuízos causados na sua formação, seja pessoal como esportiva.

A partir destes aspectos, o objetivo deste estudo foi analisar os níveis de QV percebidos por atletas de voleibol feminino de base em diferentes momentos do período de afastamento social e compará-los nas dimensões física, psicológica, social e ambiental. 


\section{METODOLOGIA:}

Este é um estudo quantitativo, descritivo e comparativo, decorrente de uma coleta de dados longitudinal. As informações tiveram origem no banco de dados de um clube de voleibol do interior do Rio Grande do Sul, que treina sistematicamente e participa de competições oficiais da Federação Gaúcha de Voleibol, Confederação Brasileira de Voleibol e Comitê Olímpico Brasileiro. O clube mantém um banco de dados sobre os treinamentos e avaliações realizadas com suas atletas e, para este estudo, disponibilizou acesso aos dados das 25 atletas das equipes de competição femininas das categorias de base, sendo 13 atletas do sub16 e 12 do sub18 anos, com média de idade de 16,2 anos. As informações coletadas datam do período de abril a agosto de 2020, representando do primeiro ao último mês do período considerado como “afastamento social" no Rio Grande do Sul, causado pela pandemia COVID19, que gerou interrupção nos treinamentos presenciais e as aulas nas escolas frequentadas por estas atletas.

Durante o período citado, as atletas mantiveram-se treinando de forma remota (online), em duas a três sessões semanais, com duração de uma hora a uma hora e meia cada, ministradas pelos técnicos, assistentes técnicos e preparador físico do clube. Nestes treinamentos foram realizados exercícios voltados ao condicionamento físico (força, resistência e mobilidade) e manutenção de habilidades técnicas de manuseio de bola (toque, manchete e cortada).

Para o presente estudo, os dados utilizados foram as avaliações que a comissão técnica realizou da QV das atletas. Para tanto, foi aplicada versão abreviada do instrumento World Health Organization Quality of Life - WHOQOL-bref (OMS, 1995), em sua versão adaptada por Rother (2017) para atletas jovens. Os níveis dos resultados foram classificados de acordo com proposto por Padrão (2008) e divididos nas dimensões de análise física, social, ambiental e psicológica, utilizadas pelo WHOQOL-bref (1995). Padrão (2008) propõe a classificação dos escores de QV como Muito Ruim (0 a 20 pontos), Ruim (21 a 40), Nem ruim nem bom (41 a 60), Bom (61 a 80) e Muito Bom (81 a 100). Além do instrumento já descrito para avaliação da QV, a comissão técnica também registrou no banco de dados as respostas de duas questões acrescentadas ao Whoq-bref sobre o tempo passado em afastamento social (em dias) e o rigor percebido deste afastamento (1-Nenhum: saio de casa e faço tudo normalmente; 2-Pouco: evito somente alguns lugares; 3-Mais ou menos: evito sair mas às vezes vou a lugares desnecessários; 4-Muito: saio de casa somente para lugares que são realmente necessários; 5-Completamente: não saio de casa).

Todos os dados foram tabulados com o auxílio de planilhas de cálculo no Excel@, quantificando os valores totais, médias, desvios padrão e percentuais. Para comparar as dimensões da QV e determinar qual delas foi a mais afetada, utilizou-se um teste de Friedman, que comparou os resultados entre as dimensões ao longo do tempo (de abril a agosto). Já as comparações “par-a-par” destas variáveis foram feitas a partir do teste de Dunn. Para comparar como os diferentes níveis de QV se comportaram ao longo do tempo, utilizou-se um teste de Kruskall-Wallis, também com comparações 'par-a-par” feitas através do teste de 
Dunn. A fim de determinar se a variação do rigor do afastamento estava relacionada com a variação da QV, utilizou-se uma regressão linear múltipla, para cada dimensão, tendo o mês e o rigor como fatores. O rigor e os meses foram transformados em escores para adequar-se à análise.

As estatísticas foram calculadas para o total de atletas e também estratificadas em dois grupos (sub16 e sub18) considerando que possíveis diferenças de idade interfiram nos resultados. Todas as análises levaram em consideração um alfa de significância de 0.05. As análises foram desenvolvidas no programa estatístico PAST 4.03. Foram tomados todos os cuidados éticos para realização deste estudo, sendo o mesmo aprovado pelo Comitê de Ética em Pesquisa da Univates - COEP, sob parecer número 1.919.927.

\section{RESULTADOS}

\subsection{QV E SUAS DIMENSÕES}

Os resultados dos escores de QV das 25 atletas avaliadas para o período de abril a agosto apresentaram média de 69,9 pontos. Quando estratificadas por idade, a média das atletas Sub16 ficou em 71,9 e das Sub18 em 67,9. O mês de abril foi o momento onde se evidenciou mais a QV das atletas Sub16 $(74,4)$, já o mês de julho foi o maior escore $(75,9)$ para o Sub18. Quanto às dimensões, a Física foi a melhor avaliada $(77,6)$ e a Psicológica a pior $(65,1)$ entre as Sub16. No Sub18 a melhor ficou com a Social $(72,1)$ e a pior também a Psicológica $(63,2)$. Os valores para cada dimensão da QV em cada mês podem ser observados na Tabela 1.

Tabela 1 - Escores de QV gerais e por dimensão para cada mês de afastamento social.

\begin{tabular}{|c|c|c|c|c|c|c|c|}
\hline & Mês & Abril & Maio & Junho & Julho & Agosto & \\
\hline Categorias & Dias afastamento & 34,2 & 66,9 & 98,2 & 127,2 & 163,8 & MÉDIA \\
\hline \multirow{6}{*}{ Sub16 } & $\mathrm{n}$ & 13 & 12 & 13 & 13 & 12 & 12,6 \\
\hline & Social & 75,6 & 66,7 & 69,9 & 69,2 & 68,1 & 69,9 \\
\hline & Físico & 77,7 & 77,1 & 77,5 & 80,2 & 75,3 & 77,6 \\
\hline & Psico & 68,9 & 64,2 & 66,3 & 64,1 & 61,8 & 65,1 \\
\hline & Ambiental & 75,5 & 78,6 & 72,6 & 73,1 & 76,0 & 75,2 \\
\hline & Geral & 74,4 & 71,7 & 71,6 & 71,7 & 70,3 & 71,9 \\
\hline \multirow{8}{*}{ Sub18 } & $\mathrm{n}$ & 12 & 13 & 12 & 10 & 13 & 12,0 \\
\hline & Social & 73,6 & 62,2 & 68,8 & 80,8 & 75,0 & 71,1 \\
\hline & Físico & 63,7 & 56,3 & 62,5 & 72,5 & 68,2 & 64,6 \\
\hline & Psico & 55,9 & 65,7 & 61,1 & 67,9 & 65,5 & 63,2 \\
\hline & Ambiental & 64,6 & 62,7 & 70,6 & 82,5 & 78,7 & 71,8 \\
\hline & Geral & 64,5 & 61,7 & 65,8 & 75,9 & 71,9 & 67,9 \\
\hline & Média & 69,4 & 66,7 & 68,6 & 73,8 & 71,1 & 69,9 \\
\hline & $\mathrm{dp}$ & 18,95 & 18,44 & 18,14 & 20,20 & 19,44 & 18,64 \\
\hline
\end{tabular}

Fonte: os autores (2021) 
Os resultados já apresentados, com as médias entre 61 e 80 pontos são classificadas por Padrão (2008) como "boas". A partir desta classificação, foi montado um mapa de radar das médias de QV para as diferentes dimensões. Embora todas sejam boas, a categoria com menor escore foi a Psicológica $(64,2)$, sendo evidenciado no mapa pelo leve desvio descendente. A dimensão com maior escore foi a Ambiental $(73,5)$, bem equilibrada com a Social $(71)$ e Física $(71,1)$. Os valores do mapa podem ser observados na Figura 1.

Figura 1 - Mapa de Radar com a classificação das dimensões de QV das atletas de voleibol feminino de base.

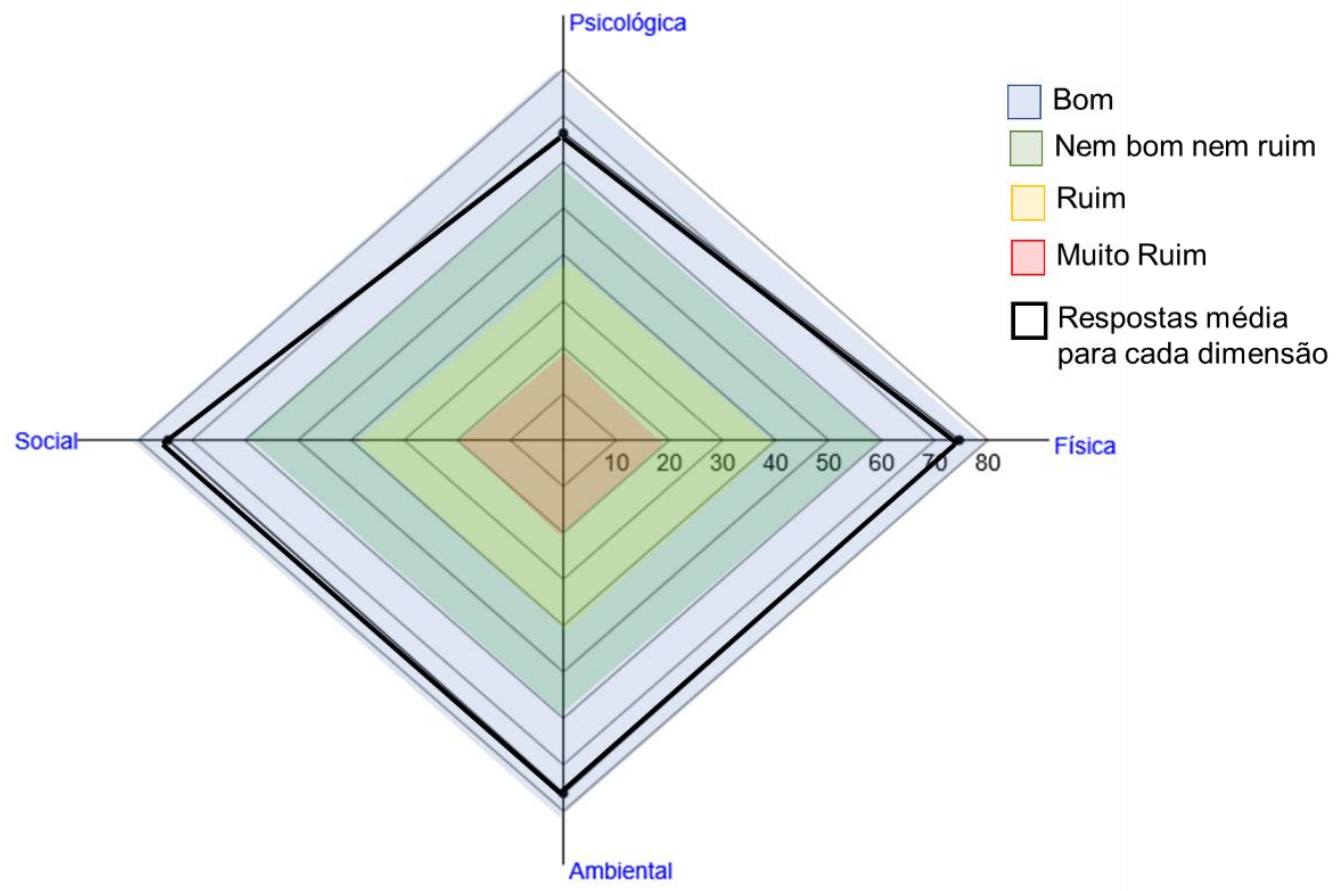

Fonte: os autores (2021).

\subsection{COMPARAÇÃO DA QV ENTRE OS MESES DE PANDEMIA}

O teste de Friedman demonstrou claras diferenças entre as dimensões estudadas ao longo do tempo $\left(\mathrm{x}^{2}=54.54 ; \mathrm{gl}=3 ; \mathrm{p}>0.0001\right)$. Como mostra o Gráfico 1, a dimensão Psicológica obteve escores estatisticamente menores que as demais dimensões, durante todo o período de afastamento, com um aumento (não significativo) em junho. 


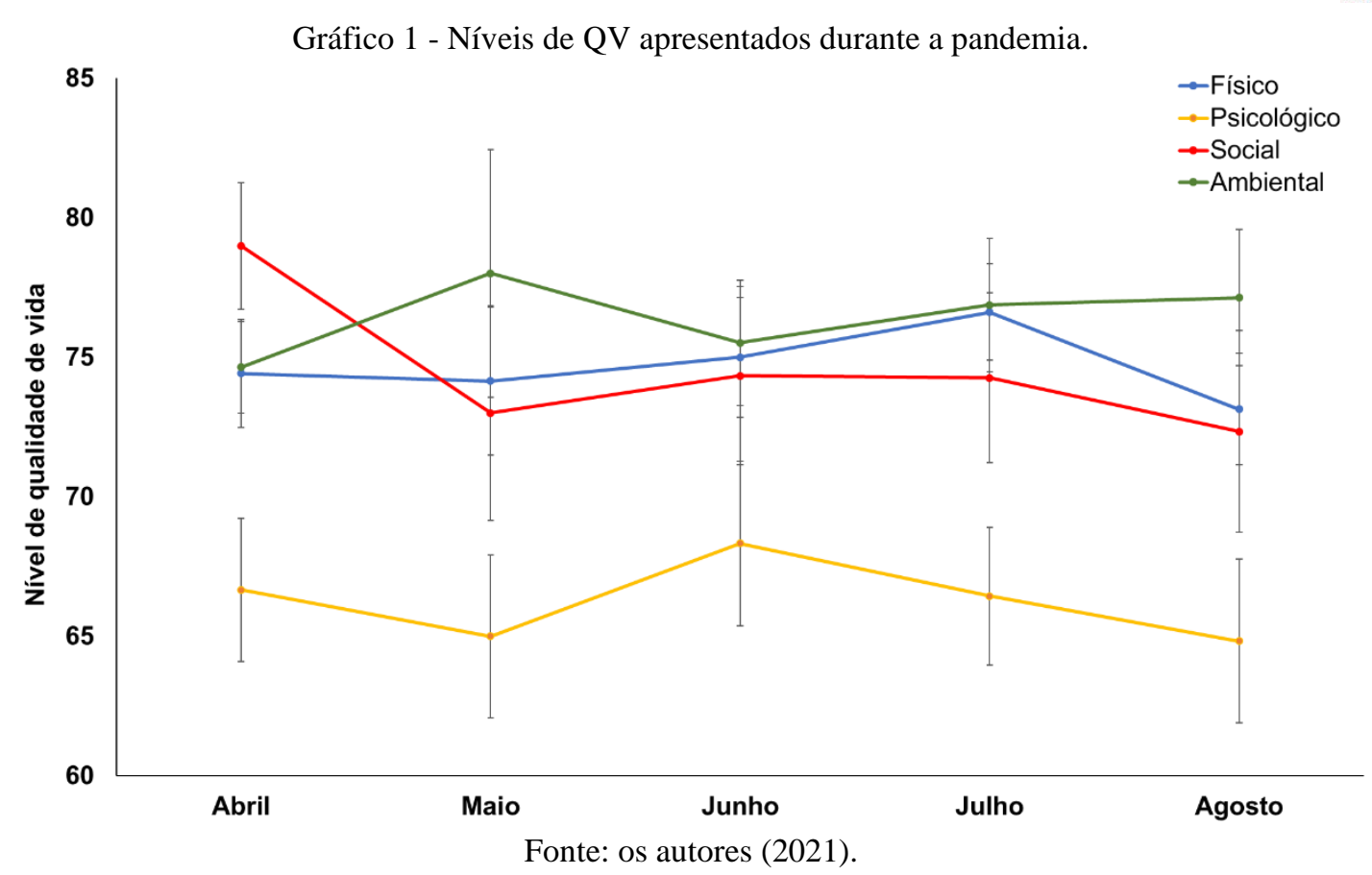

As demais dimensões não foram diferentes entre si e não variaram ao longo do tempo, embora a dimensão Social tenha apresentado uma queda considerável a partir de maio. Estatisticamente, não houve variação na $Q V$ ao longo do tempo observado (Geral $p=0.951$; Físico $p=0.820$; Psicológico $p=0.900$; Social $\mathrm{p}=0.506$; Ambiental $\mathrm{p}=0.955)$, somente uma diferença significativa na dimensão Física $(\mathrm{p}=0.002)$ maior nas atletas Sub16 e na Social $(\mathrm{p}=0.001)$ maior na Sub18.

\subsection{TEMPO E RIGOR DO AFASTAMENTO SOCIAL}

As 25 atletas apresentaram uma média de 163,8 dias de afastamento social, caracterizado pelo tempo de paralisação dos treinamentos e aulas presenciais, em que mantiveram-se reclusas em suas casas. As atletas classificaram em uma escala de 1 a 5 o rigor do afastamento adotado, tendo as Sub16 e Sub18 respondido, respectivamente, em abril (3,7 e 3,7), maio (4,0 e 4,0), junho (3,7 e 3,3), julho (3,6 e 2,8) e agosto $(3,5$ e 3,3$)$.

Quando comparadas entre as atletas de maior e menor idade, estas respostas mostraram-se estatisticamente diferentes ( $\mathrm{p}=0.02$ ), sendo mais rigoroso o afastamento na categoria Sub16, como mostra o Gráfico 2. Além disso, quando analisado o rigor ao longo dos meses, percebe-se que as atletas mais jovens não apresentaram variação ( $\mathrm{p}=0.40)$, diferentemente do que ocorreu com as atletas mais velhas $(\mathrm{p}=0.002)$. 
Gráfico 2 - Rigor do afastamento social estratificado por idade.

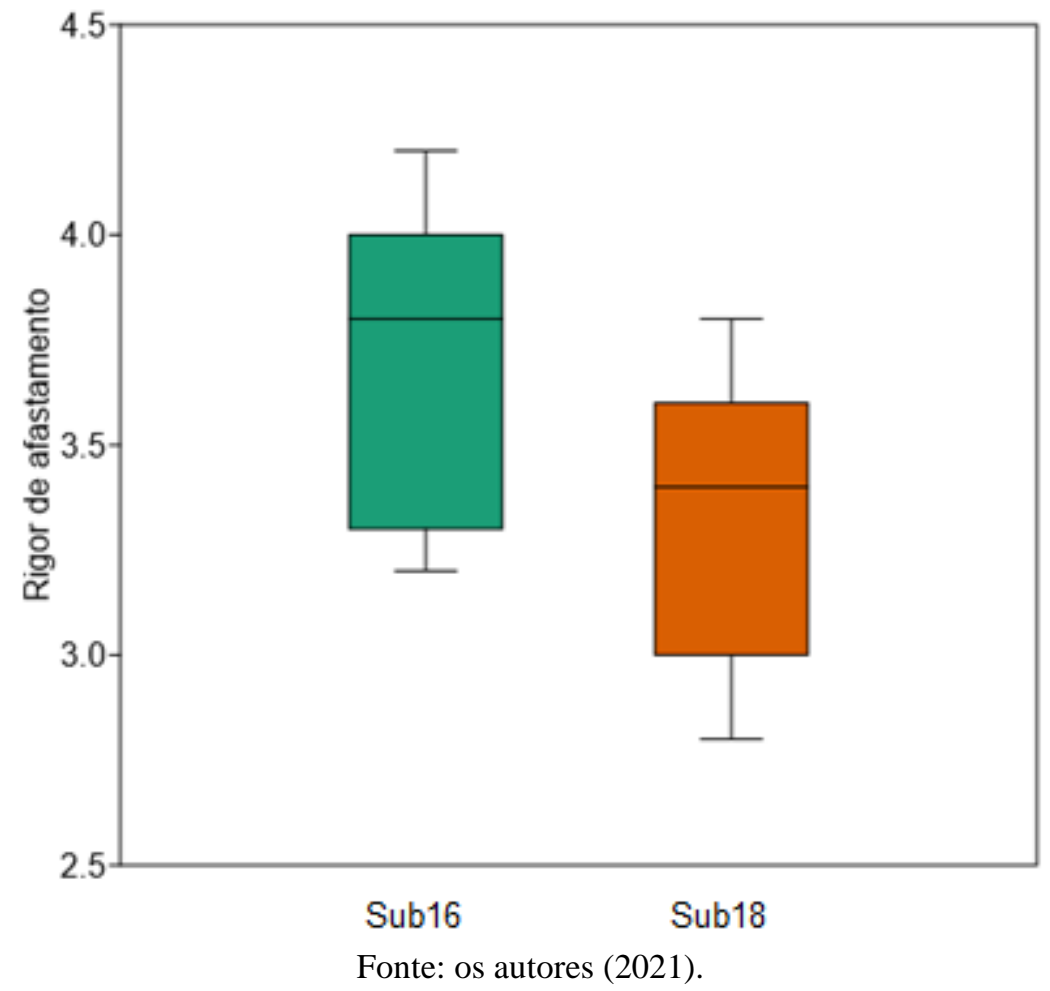

$\mathrm{Na}$ busca de uma possível correlação entre o rigor do afastamento e os escores de QV durante o período, as análises de regressão indicaram ser significativo na dimensão Física (p=0.049) e Geral $(\mathrm{p}=0.044)$. As demais dimensões não foram influenciadas, seja quando observadas para o total de atletas, seja quanto estratificado em categorias por idade.

\section{DISCUSSÃO}

Mesmo com a pandemia interferindo em todas as dimensões da vida das atletas, mudando suas rotinas e às impedindo de realizar muitas das atividades que estão habituadas e conviverem presencialmente nos diversos ambientes que frequentavam costumeiramente, ainda assim percebeu-se que os escores de QV indicam classificação boa.

Estudos realizados em períodos onde não havia afastamento social, anteriores à pandemia, indicaram escores de valor um pouco superiores aos aqui encontrados mas não o suficiente para serem classificados como nível superior ao "Bom”. Rother e Silva (2017) avaliaram a QV de 36 atletas de voleibol feminino escolar de 12 á 17 anos e encontraram resultados para as dimensões Física $(73,43 \pm 12,92)$, Psicológica $(70,97 \pm 12,02)$ e Ambiental $(73,98 \pm 8,62)$ classificados como bons e Social $(81,94 \pm 11,69)$ como muito bom. Já Schinader e Rother (2020) avaliaram a QV de 28 atletas de voleibol feminino, de 14 a 17 anos, comparando a QV e o rendimento esportivo entre as que vivem longe de seus familiares (G1) e as que vivem em casa com seus pais (G2). Os resultados encontrados para cada dimensão foram: Física (G1 


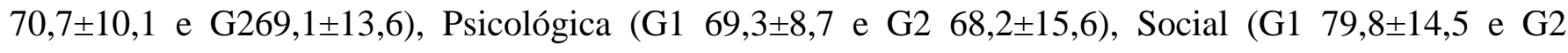
79,8 $\pm 12,1$ ), Ambiental (G1 73 $\pm 8,9$ e G2 74,6 $\pm 8,1$ ) e Geral (G1 73,2 $\pm 4,6$ e G2 72,93 $\pm 5,4$ ). Ambos os grupos foram classificados como bons níveis de QV e não foi encontrada diferença significativa entre eles.

Como pode ser visto em ambos os estudos, mesmo sendo considerada boa, a dimensão Psicológica foi a que obteve os menores escores, corroborando com os resultados encontrados no presente estudo. Isso pode justificar-se pela mudança, não somente na rotina dessas atletas, mas também pela incerteza de seus futuros, já que muitas sonham em jogar profissionalmente e a pausa nas competições as afasta de inúmeras oportunidades para isso. As atletas também têm muitas das suas conexões facilitadas pela prática esportiva ou treinamento e a cessação deste contato pode causar prejuízos e efeitos no bem-estar subjetivo (HUREMOVIC, 2019). Em estudo recente, Brooks et al. (2019) listaram causas de transtornos psicológicos negativos que acometem as pessoas durante a pandemia, os quais também podem interferir nos escores de QV percebidos pelas atletas aqui investigadas, são eles: estresse, medo de infecção, frustração, suprimentos insuficientes, informações confusas ou insuficientes e perdas financeiras.

É importante salientar ainda que as atletas continuaram a treinar de forma remota durante o afastamento social e os benefícios da prática de exercício físico podem ter ajudado a não deixar este resultado cair ainda mais, outro benefício que ajudou a não cair significativamente os nívies de QV foi o suporte da família, dos treinadores, professors da escola, grupos de conversa via redes sociais, o que manteve o espírito de equipe forte e a esperança de que quando a pandemia passar seus lugares na equipe e no colégio estão garantidos, podendo assim retornar a sua vida normal. Para exemplificar esta afirmação, Pitanga et al. (2019) encontraram em seus estudos relações entre a prática de exercício físico crônica e a minimização dos sintomas de ansiedade e depressão. O esporte muitas vezes é utilizado para distração e para aliviar as tensões do cotidiano, o que pode auxiliar na manutenção de bons níveis de QV.

Quanto à dimensão Social, os estudos já citados (ROTHER; SILVA, 2017; SCHINADER; ROTHER, 2020) apresentaram escores mais altos para esta dimensão quando comparadas às outras dimensões da QV, enquanto os resultados aqui encontrados apresentam a Social como a segunda mais baixa. Martins et al. (2002) estudaram o esporte escolar e ressaltaram que, além de aprenderem sobre regras de convivência, nas interações entre crianças e adolescentes ocorre o desenvolvimento da percepção de mundo e um exercício de identificação dos seus papéis sociais. Estes autores afirmam ainda que é pelo esporte que as crianças e adolescentes aprendem a conviver com vitórias e derrotas e vencerem através do seu esforço.

A variável "pandemia" pode ser considerada aqui uma importante agente de influência neste resultado pela imensa mudança ocorrida na rotina destas atletas, não se referindo apenas aos treinamentos e viagens para jogos, mas também por não realizar de forma presencial quase nenhuma das atividades costumeiras, como ir a escola, conversar diariamente com as colegas, amigos e familiares. Ainda que, 
quando avaliado todo o grupo de atletas a QV Social tenha sido a segunda pior, quando considerada somente as atletas mais velhas (Sub18), o rigor do afastamento social adotado foi menor e os escores da QV Social foram maiores que as atletas mais jovens, o que pode ser um indicador que corrobora com os dados apresentados na literatura sobre a relação das interações sociais e a QV Social (OMS, 1995).

Sobre a dimensão Física da QV, ao final do estudo, foram confirmadas correlações entre os níveis de QV Geral e Física, demonstrando impacto prejudicial do rigor do afastamento social nestas duas variáveis estudadas. Ainda assim, os níveis de QV foram classificados como bons e conseguiram ser mantidos durante todo o período investigado. Reforça-se que as atletas analisadas neste estudo continuaram a treinar de forma remota, com orientação profissional, em suas casas, do início ao final do período de afastamento social.

Sobre as relações da prática esportiva com os aspectos físicos, Nieman (1994) já afirmava haver associação com a melhora do sistema imunológico. Corte et al (2020) alertam para a fragilização que o sistema imunológico pode sofrer pela redução da regularidade do exercício físico e a vulnerabilidade a contrair e desenvolver infecções ou outras patologias. Além disso, os autores lembram também que, a partir da redução de treinamentos usualmente utilizados na prevenção de lesões, o risco de ocorrência das mesmas aumentam significativamente. $\mathrm{O}$ treinamento físico crônico de intensidade moderada tem relação direta com menor índice, duração e gravidade de infecções no trato respiratório superior que são predominantemente virais, reforçando a importância do exercício físico para a melhora do sistema imunológico, já citado anteriormente (NIEMAN; WENTZ, 2019). Além disso, considerando os benefícios a longo prazo, a atividade física auxilia no controle da hipertensão arterial, diminuição dos níveis de obesidade, sarcopenia, entre outras (INÁCIO et al., 2021).

Ainda como benefícios gerais da prática esportiva nos aspectos físicos, Tubino (1999) classifica o esporte como uma manifestação da cultura física, que possibilita ao indivíduo desenvolver as capacidades física, motora, cognitiva, respeito e a socialização. Já Rother e Silva (2017), reforçam os benefícios desta prática para a $\mathrm{QV}$, mas ressaltam que treinar em volumes elevados pode ter um efeito inverso, devendo-se ter cuidados com o controle das cargas, principalmente em atletas jovens.

A manutenção da prática esportiva foi um grande desafio para os profissionais responsáveis por planejar, aplicar e avaliar o treinamento durante a pandemia. Segundo o Conselho Regional de Educação Física do Estado de São Paulo - CREF4/SP (2020), mesmo dentro de casa é importante que o atleta vivencie, sinta corporalmente os benefícios da realização dos exercícios. Pensando nisso, prescreve-se o treinamento de acordo com os espaços, recursos comuns e materiais adaptáveis disponíveis em casa. O uso de tecnologias foi fundamental neste processo, ainda mais quando utilizadas de forma online. Certamente o período marcou uma transformação na atuação do profissional desta área e possibilitou a criação de novas ferramentas e formas pedagógicas de ensino/treinamento (CREF4/SP, 2020). 
Pelo lado das atletas, a partir da necessidade de permanecerem em suas casas e realizarem todas as atividades neste local, as famílias obrigaram-se a estruturar todo um ambiente de suporte necessário para um estudo e treinamento de qualidade. A pandemia também interferiu na situação financeira de muitas famílias e ter condições para melhorar o acesso à internet de qualidade e adquirir equipamentos tecnológicos mais atuais não foi possível para todos, mas devemos salientar que o treinador e a equipe responsável sempre deu todo suporte necessário diariamente além de conversas em grupos de mensagens mantendo a atletas sempre focadas e com motivação par treinarem, fazendo todo possível para fazer do treino mais proveitoso possível com o ambiente onde vivem. Este aspecto, embora não tenha sido avaliado profundamente neste estudo, merece uma atenção pois pode ser um limitador no dia a dia das atletas, interferindo negativamente na sua QV. Lembrando que a dimensão Ambiental da QV se dá pelo juízo de valor que o sujeito percebe para as condições onde vive, suas necessidades, desejos e a realização dos mesmos (BARBOSA, 1998).

\section{CONCLUSÃO}

Os escores de QV das atletas não apresentaram diferença significativa ao longo do tempo de afastamento social, mantendo-se bons e estáveis. O tempo do afastamento parece não ter impactado na QV, mas sim o rigor, tanto na QV Geral, quanto na dimensão Física especificamente. O nível de rigor no afastamento social adotado e a continuidade na realização de treinamentos online durante o período podem ser variáveis que auxiliaram na manutenção de níveis bons de QV. Mais estudos sobre os efeitos da prática de treinamentos em casa merecem atenção, já que foi uma prática comum para atletas de várias modalidades esportivas durante a pandemia. 


\section{REFERÊNCIAS}

BARBOSA, S.R.S. Qualidade de Vida e ambiente: uma temática em construção. In: BARBOSA, S.R.S. (org.). A temática ambiental e a pluralidade do Ciclo de Seminários do NEPAM. Campinas: UNICAMP, NEPAM, 1998, p. 401-423.

BENETTI, G.; SCHNEIDER, P.; MEYER, F. Os benefícios do esporte e a importância da treinabilidade da força muscular de pré-púberes atletas de voleibol. Rev. Bras. Cineantropom. Desempenho. Hum, v. 7, n. 2, p. 87-93, 2005.

BROOKS, S.K.; WEBSTER, R.K.; SMITH, L.E.; WOODLAND, L.; WESSELY, S.; GREENBERG, N.; et al. The psychological impact of quarantine and how to reduce it: rapid review of the evidence. The Lancet. 2020, v. 395 (2): 912-20.

CARAZZATO, J.G. A criança e o esporte: idade ideal para o início da prática esportiva competitiva. Rev Bras Med Esporte. 1995, (1): 97-101.

CORTE, A.C.R.; CAMARGO-JÚNIOR, F.; CHALHUB, T.; MORAES FILHO, J.M.; SANTOS, R.; FEITOSA, F.; et al. COVID-19 e seu efeito no esporte olímpico: a importância de estudar o isolamento social e seus danos a fim de minimizá-los. Rev Bras Med Esporte, São Paulo, v.26, n.5, Oct. 2020, p.371-77.

CONSELHO REGIONAL DE EDUCAÇÃO FÍSICA DE SÃO PAULO - CREF4/SP. Boletim COVID-19: Recomendações da Educação Física Frente à Pandemia por COVID 19. São Paulo, CREF: 2020.

HUREMOVIC, D. Psychiatry of Pandemics: A Mental Health Response to Infection Outbreak. Gewerbestrasse: Springer Nature, 2019.

INÁCIO, G. P. ;; FONTANA, A. P. .; PEIXOTO, D. B. .; MACHADO, A. V. .; XAVIER, F. D. S. .; MENEZES, M. S. .; AQUINO, M. A. R. .; INÁCIO, J. P. . A importância da atividade física e alimentação na hipertensão arterial. Archives of Health, v. 2, n. 2, p. 166-170, 4 May 2021.

MOURA, D.L.; DIAS, A.; TORRES, J.P.; FARINHA, P.; RIBEIRO, B.; ROBALO-CORDEIRO, C. Pandemia COVID-19 e impacto no desporto. Rev. Medicina Desportiva informa, 2020; 11(3): 26-33.

MARTINS, D.F.; REZER, R.; CASTRO, R.L.V.G.; SHIGUNOV, V. O esporte como papel de uma reunião social. RECE, 2002, v.1, (1): 135-46.

NIEMAN, D.C. Exercise, infection and immunity. Int J Sports Med. 1994; 15 (3): p.131-41.

NIEMAN, D.C.; WENTZ, L.M. The Compelling link between physical activity and the body's defense system. J Sport Health Sci. 2019, v.8 (3): 201-217.

ORGANIZAÇÃO MUNDIAL DA SAÚDE (OMS). The World Health Organization Quality of Life Assessment (WHOQOL): position paper from the World Health Organization. Social science and medicine. v.41, n.10, 1995, p.403-409.

ORGANIZAÇÃO MUNDIAL DA SAÚDE (OMS). Folha informativa COVID-19. Versão 5, dezembro 2020. 
ORGANIZAÇÃO PANAMERICANA DE SAÚDE (OPAS). Orientações técnicas da OPAS/OMS sobre COVID-19 para profissionais da saúde. Perguntas frequentes sobre as vacinas candidatas contra a COVID-19 e os mecanismos de acesso. Versão 2, agosto 2020.

PADRÃO, M.B. Avaliação da qualidade de vida de doadores vivos após o transplante renal utilizando os instrumentos SF - 36 e WHOQOL- bref. Dissertação Mestrado. Faculdade de Ciências Médicas, São Paulo/SP. 2008.

ROTHER, R.L. Desempenho esportivo de atletas infantis do voleibol gaúcho e as relações com a qualidade de vida. 2017. Monografia (Doutorado) - Curso de Ambiente e Desenvolvimento, Universidade do Vale do Taquari - Univates, Lajeado, 20 out. 2017.

SCHINADER, B.; ROTHER, R.L. Percepção da Qualidade de Vida e do rendimento esportivo de atletas que vivem longe de suas famílias, Braz. J. of Develop., Curitiba, v. 6, n. 6, p. 35294-35306, jun. 2020.

ROTHER, R.L.; SILVA, S.S.M. Percepção da qualidade de vida de atletas femininas de voleibol escolar em diferentes categorias 2017, CINERGIS. Santa Cruz do Sul, Unisc, Ano 18 - Volume 18 - Número 3 Julho/Setembro 2017.

SIMÕES, C.S.M. Análise das percepções da Qualidade de Vida: do estresse e da recuperação de atletas de voleibol de diferentes categorias. Belo Horizonte/MG: Universidade Federal de Minas Gerais. Escolas de Educação Física, 2011.

TENFORDE, M.; KIM, S.S.; LINDSELL, C.; ROSE, E.B.; et al. Symptom Duration and Risk Factors for Delayed Return to Usual Health Among Outpatients with COVID-19 in a Multistate Health Care Systems Network. MMWR - Morbidity and mortality weekly report. Julio, 2020: 69(30).

TUBINO M. O que é esporte: uma enciclopédia crítica. 2 Ed. Vol. 276. São Paulo: Brasiliense, 1999.

ZHU, W. Should, and how can, exercise be done during a coronavirus outbreak? An interview with Dr. Jeffrey A. Woods. Journal of Sport and Health Science. 2020, 9(2), 105-107. 